\title{
A comprehensive review of available omics data resources and molecular profiling for precision glioma studies (Review)
}

\author{
${\text { ZHENG } \text { ZHAO }^{1 *}, \text { KENAN ZHANG }^{1,2^{*}}, \text { ZHILIANG WANG }}^{1,2}$, \\ KUANYU WANG $^{1,2}$, XING LIU ${ }^{1}$, FAN WU ${ }^{1}$ and JING CHEN ${ }^{1}$ \\ ${ }^{1}$ Department of Molecular Neuropathology, Beijing Neurosurgical Institute, Capital Medical University, Beijing 100050; \\ ${ }^{2}$ Department of Neurosurgery, Beijing Tiantan Hospital, Capital Medical University, Beijing 100050, P.R. China
}

Received April 4, 2018; Accepted November 11, 2018

DOI: $10.3892 /$ br.2018.1168

\begin{abstract}
Gliomas are the most common and lethal type of primary malignant central nervous system tumors, with an extremely poor prognosis. The latest progression in the technological development of sequencing/microarray and bioinformatics has provided insights into the glioma genome. These technologies have generated large amounts of easily accessible biological omics data, providing an unprecedented opportunity to study glioma formation. According to the 2016 WHO organization classification of brain tumors, gliomas are currently diagnosed with respect to morphological and molecular tumor alterations, especially for isocitrate dehydrogenase and $1 \mathrm{p} / 19 \mathrm{q}$ codeletions. In the present study, the comprehensive molecular profiling and available omics data resources for malignant gliomas were reviewed for novel insights into the biology and classification of these tumors. These molecular profiling resources may be useful for improving the understanding of malignant gliomas, and to accelerate the clinical, experimental and epidemiological studies that may lead to improvements in the lives of patients with glioma.
\end{abstract}

\section{Contents}

1. Introduction

2. Available glioma data resources

3. Molecular profiling as a precise clinical tool

4. Conclusions

Correspondence to: Dr Jing Chen, Department of Molecular Neuropathology, Beijing Neurosurgical Institute, Capital Medical University, 6 Tiantan Xili, Dongcheng, Beijing 100050, P.R. China E-mail: chenjing0405@126.com

*Contributed equally

Key words: glioma, omics data, molecular profiling

\section{Introduction}

Gliomas are the most common primary and among the most fatal types of malignant brain tumors, with an annual incidence of 5.26 per 100,000 population and an overall 17,000 incident diagnoses per year in the United States; and the number of patients is expected to increase with increases in population age (1-3). Based on the histopathological classification of the 2016 WHO central nervous system tumors, gliomas are diagnosed from lowest to highest grades: Grade I angiocentric gliomas; grade II oligodendrogliomas [isocitrate dehydrogenase (IDH)-mutant and 1p/19q-codeleted] and diffuse astrocytomas (IDH-mutant); grade III anaplastic oligodendrogliomas (IDH-mutant and 1p/19q-codeleted) and anaplastic astrocytomas (IDH-mutant); and finally grade IV glioblastomas (GBM) and diffuse midline gliomas (H3K27m-mutant) (4). Although histopathological grades remain useful, the prognoses of patients with glioma are more closely associated with molecular alterations compared with grades $(5,6)$. Notably, patients with glioma are typically associated with heterogeneous tumor morphologies and variable prognoses. Despite therapeutic advances, gliomas remain incurable, with the most aggressive forms resulting in mortality within months (7). Therefore, there is an urgent requirement to increase the understanding of the etiology, pathology and taxonomy of glioma, to improve the selection of treatment options for patients and to develop novel therapeutic strategies.

High-throughput sequencing and microarray technologies, for example whole-genome sequencing and RNA sequencing, provide an opportunity to examine the broad range of genomic information at an unprecedented high resolution. Previous technological advances (e.g., RNA-Seq, microarray) and the exponential decrease in the cost of genome sequencing and microarray detection have made biological data resources increasingly accessible (8-10). In previous decades, molecular biomarker-based diagnostics with glioma have resulted in a change in the traditional pathological classification methods of glioma, resulting in more reproducible and precise clinically distinct subtypes (11-15). At present, IDH mutations and codeletion of chromosome $1 p$ and $19 q$ arms, as a molecular signature, are now included in standard glioma diagnosis and classification methods (4,16-18). In addition, the roles of several key genes have been described, including telomerase 
reverse transcriptase (TERT) $(19,20)$, phosphatase and tensin homolog (PTEN) (21), alpha thalassemia/mental retardation syndrome X-linked (ATRX) (22), B-Raf proto-oncogene, serine/threonine kinase (23) and O-6-methylguanine DNA methyltransferase (MGMT) (24). In light of these data, the molecular genetic signatures should be valuable in the diagnostic evaluation or treatment strategies for glioma (25).

Large population-based data sources are required to provide accurate descriptions of the mechanism of glioma. Previous data collection and integrative data analyses, for example, The Cancer Genome Atlas (http://cancergenome.nih. gov/) or Chinese Glioma Genome Atlas (http://cgga.org.cn), have revealed the genomic landscape of either low-grade or high-grade gliomas. In the present review, the available data resources and the molecular biomarkers from precision glioma studies were summarized. The aim of the present study was to provide a wide overview of existing data and recent molecular profiling, which may improve the current knowledge base in this field, and thereby assist in generating novel pathological classification categories, diagnostic methods, and novel therapeutic approaches to accelerate studies into the causes and control of glioma.

\section{Available glioma data resources}

Previously described high-throughput technologies have provided the opportunity for the extensive characterization of genomic statuses, including, but not limited to, genetic alterations, methylation modification and gene expression regulation $(26,27)$. Over the previous decade, several genome projects were initiated, accelerating the comprehensive understanding of the genetics of glioma (Table I). Using innovative genome analysis technologies may assist in generating novel glioma therapies, diagnostic methods and preventive strategies $(12,28,29)$. Therefore, the present study reviewed currently available glioma data resource in this community.

The Cancer Genome Atlas (TCGA). In 2005, the National Institute of Health and National Human Genome Research Institute began the TCGA project to generate comprehensive multi-dimensional maps of the key genomic alternations that promote malignant transformation. TCGA pilot project aimed to depict the molecular characteristics of multiple 'omics' in human cancer and to generate a data resource for the scientific community. As the most common and lethal intracranial tumor, glioma was the first type of cancer studied by TCGA Research Network. At present, it contains data from GBM and lower grade gliomas from multiple platforms, including copy number (1,090 samples), DNA methylation (936 samples), RNA-seq profiling (676 samples), mRNA microarray profiling (567 samples), miRNA microarray profiling (565 samples) data recorded in the Broad FireBrowse database (http://firebrowse.org/). Integrative analysis of DNA copy number, gene expression and DNA methylation data in 206 GBMs revealed that several key genes [including erb-B2 receptor tyrosine kinase 2, neurofibromin 1 (NF1), tumor protein p53 (TP53), phosphoinositide 3 -kinase regulatory subunit 1 and cyclin dependent kinase inhibitor 2A/B (CDKN2A/B)] and signaling pathways (including receptor tyrosine kinase/Ras/phosphoinositide 3-kinase, TP53 and CDKN2A) were identified to be frequently mutated in human GBMs, demonstrating that these data may improve the understanding of the molecular basis of cancer (30). In addition, a robust gene expression-based molecular classification of GBMs into the proneural, neural, classical and mesenchymal subtypes described by TCGA network provided evidence to support the requirement for targeted therapeutics (13). This study unifies genomic and transcriptome dimensions for molecular GBM stratification by abnormalities in platelet derived growth factor receptor alpha (PDGFRA), isocitrate dehydrogenase (NADP $(+)) 1$, cytosolic (IDH1), epidermal growth factor receptor (EGFR) and NF1 genes, which may provide important insight for future clinical application. Previously, Brennan et al (28) performed the largest multi-platform genomic analysis to define the critical genes associated with gliomas by using 1,122 all-grade gliomas from TCGA. In this study, they identified six methylation groups and four RNA expression groups, calculated molecular correlations and provided understanding of the malignant progression of gliomas. The results of the whole-genome sequencing revealed that ATRX but not TERT promoter mutations were associated with increased telomere length, indicating an alternative mechanism for telomeres lengthening. Notably, a group of IDH mutant glioma was associated with DNA demethylation and relatively poor survival; a subtype of IDH-wild glioma exhibited molecular similarity to pilocytic astrocytoma and favorable outcomes. This multi-omics glioma analysis provided novel insights into genomic alternations, emphasized the relevance of DNA methylation profiles for clinical classification, and associated somatic alterations involved in telomere maintenance.

At present, several exploratory analysis tools and databases have also been developed based on TCGA datasets. The cBio Cancer Genomics Portal (cBioPortal; http://cbioportal.org), an open-access and open-source resource, was developed as an interactive exploration of cancer genomics datasets and an intuitive method of presenting data, including the capacity to quickly view genomic alterations of genes or pathways of interest across a set of patients, and performing survival and biological network analysis $(31,32)$. The portal currently stores DNA copy number variants, methylation, mRNA and microRNA expression, protein and clinical data. In addition, these TCGA datasets may be also easily and directly downloaded via the Broad FireBrowse website, which is updated regularly. These tools provide rapid, intuitive access to cancer genomics profiling and matched clinical data, and allows the translation of these valuable data into biological insights and clinical applications. To investigate the biological nature of TCGA long non-coding RNAs (lncRNAs), Cerami et al (31) and Gao et al (32) developed the TANRIC and Co-LncRNA databases, respectively, which facilitated the study of the biological functions of lncRNA and their clinical applications.

Chinese Glioma Genome Atlas (CGGA). The CGGA project (http://cgga.org.cn) is hosted and supported by the Beijing Neurosurgical Institute and the Chinese Glioma Cooperative Group Research Network. This project is a comprehensive and coordinated effort to accelerate the understanding of the molecular basis of glioma, particularly in secondary GBMs through the application of high-throughput biotechnologies and bioinformatics. The project aims to catalogue and identify 
Table I. Summary of available omics glioma data reviewed in the present study.

\begin{tabular}{|c|c|c|c|c|c|c|}
\hline Source & Platform & $\begin{array}{c}\text { Samples } \\
\text { (n) }\end{array}$ & Histology & Grade & Survival & Reference \\
\hline \multirow[t]{4}{*}{ TCGA } & Illumina Human Methylation 27K/450K & 936 & $\mathrm{Y}$ & $\mathrm{Y}$ & $\mathrm{Y}$ & http://firebrowse.org \\
\hline & RNA-seq Hiseq & 676 & $\mathrm{Y}$ & $\mathrm{Y}$ & $\mathrm{Y}$ & http://firebrowse.org \\
\hline & mRNA Agilent-4502A/Affymetrix UG133A & 567 & Y & Y & Y & http://firebrowse.org \\
\hline & Human miRNA 8x15K Microarray & 565 & $\mathrm{Y}$ & $\mathrm{Y}$ & $\mathrm{Y}$ & http://firebrowse.org \\
\hline \multirow[t]{4}{*}{ CGGA } & Illumina Human Methylation 27K & 151 & Y & Y & Y & http://cgga.org.cn \\
\hline & RNA-seq Hiseq 2000 & 325 & $\mathrm{Y}$ & $\mathrm{Y}$ & $\mathrm{Y}$ & http://cgga.org.cn \\
\hline & mRNA Agilent Whole Human Genome & 301 & $\mathrm{Y}$ & $\mathrm{Y}$ & $\mathrm{Y}$ & http://cgga.org.cn \\
\hline & miRNA Human v2.0 Expression BeadChip & 198 & $\mathrm{Y}$ & $\mathrm{Y}$ & $\mathrm{Y}$ & http://cgga.org.cn \\
\hline Madhavan et al & mRNA Affymetrix HG-UG133 Plus 2.0 & 566 & $\mathrm{Y}$ & $\mathrm{Y}$ & $\mathrm{Y}$ & $(35)$ \\
\hline Ballester et al & Genomic Variants-next-generation sequencing & 342 & $\mathrm{Y}$ & $\mathrm{Y}$ & $\mathrm{N}$ & $(36)$ \\
\hline Gravendeel et al & mRNA Affymetrix HG-UG133 Plus 2.0 & 276 & $\mathrm{Y}$ & Y & $\mathrm{Y}$ & $(5)$ \\
\hline Lee $e t a l$ & mRNA Affymetrix HG-U133A & 191 & $\mathrm{~N}$ & $\mathrm{~N}$ & $\mathrm{~N}$ & $(53)$ \\
\hline Phillips et al & mRNA Affymetrix HG-U133A & 100 & $\mathrm{Y}$ & $\mathrm{Y}$ & $\mathrm{Y}$ & $(54)$ \\
\hline
\end{tabular}

TCGA, The Cancer Genome Atlas; CGCA, Chinese Glioma Genome Atlas; Y, yes; N, no.

Table II. Summary of key gene alternations in gliomas.

\begin{tabular}{lcc}
\hline Histology & Molecular abnormalities & WHO grade \\
\hline Oligodendroglioma & IDH-mutant \& 1p/19q-codeleted & WHO II \\
Astrocytoma & TERT promoter-wildtype & WHO II \\
(IDH-mutant) & IDH-mutant & WHO II \\
Astrocytoma & IDH-wildtype & \\
(IDH-wildtype) & IDH-mutant \\
Anaplastic astrocytoma & & WHO III \\
(IDH-mutant) & IDH-wildtype \\
Anaplastic astrocytoma & & WHO III \\
(IDH-wildtype) & TERT promoter-mutant \\
GBM & IDH-wildtype \\
(IDH-wildtype) & TERT promoter-mutant \\
GBM (IDH-mutant) & IDH-mutant \\
GBM (F3-T3+) & FGFR3-TACC3 gene fusion & WHO IV \\
Secondary GBM (ZM+) & PTPRZ1-MET gene fusion & WHO IV \\
\hline
\end{tabular}

WHO, World Health Organization; GMB, glioblastoma; IDH, isocitrate dehydrogenase; TERT, telomerase reverse transcriptase; FGFR, fibroblast growth factor receptor; TACC, transforming acidic coiled-coil containing protein; PTPRZ1, protein tyrosine phosphatase receptor type Z1; MET, MET proto-oncogene, receptor tyrosine kinase; F3-T3, FGFR3-TACC3; ZM, PTPRZ1-MET fusion.

major genomic alternations that drive glioma progression, and to provide a detailed genomic characterization of a large cohort of Chinese gliomas.

To share this resource, the CGGA portal was established as an open-access platform for the interactive exploration of multidimensional glioma genomics datasets. At present, it provides access to DNA methylation microarray (149 samples), mRNA microarray (305 samples) and sequencing (325 samples), microRNA microarray (198 samples) data and matched clinical data. Using the gene expression profile from an Agilent microarray of 225 samples from the CGGA, consensus average linkage clustering identified three major subgroups (G1, G2 and G3) (33). The G1 subtype demonstrated improved clinical outcome, young age and a high frequency of IDH1 mutation. The G3 subtype was characterized by poorer clinical outcome, older age and low frequency of IDH1 mutation. The parameters for clinical outcome, age and IDH1 mutation in the G2 subgroup were in between the 


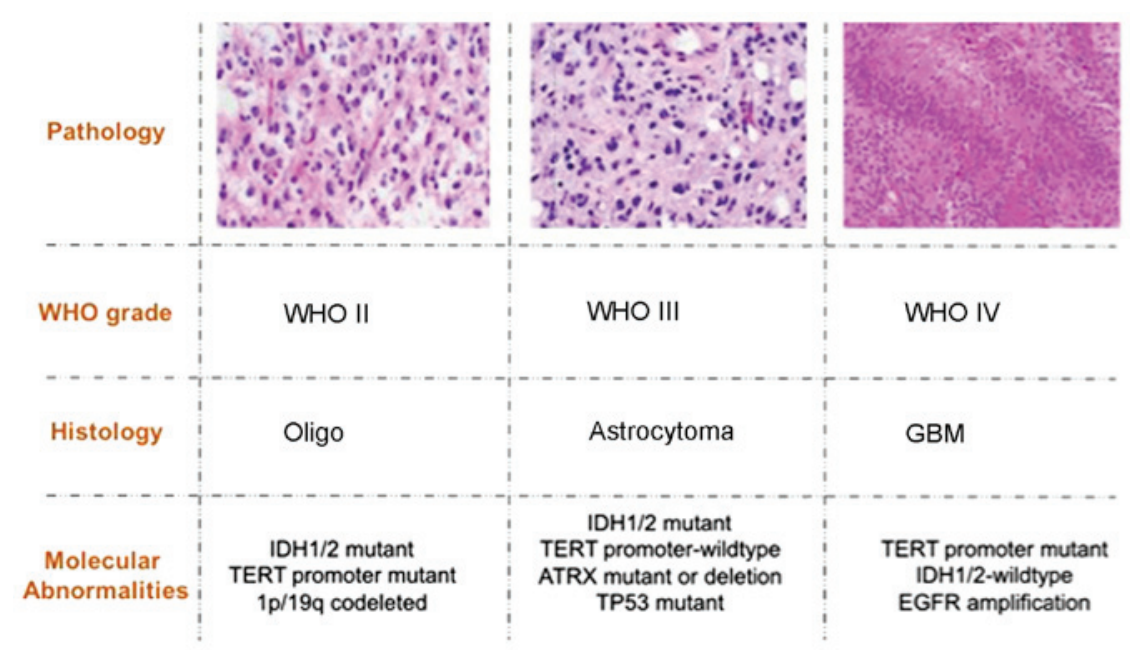

Figure 1. Traditional molecular pathology of glioma. WHO, World Health Organization; GMB, glioblastoma; IDH, isocitrate dehydrogenase; TERT, telomerase reverse transcriptase; ATRX, $\alpha$ thalassemia/mental retardation syndrome X-linked; TP53, tumor protein p53; EGFR, epidermal growth factor receptor.

values for the $\mathrm{G} 1$ and $\mathrm{G} 3$ subtypes. Combining mutation data of the TERT promoter and IDH from 377 CGGA grade II/ III glioma samples, Brat et al (12) performed a molecular classification of glioma into IDH-mutation/TERTp-mutation, IDH-mutation only, TERTp-mutation only and IDH-wild type/TERT promoter-wild type groups. Patients with only TERTp-mut genotypes exhibited the poorest prognoses, while patients with an IDH mutation alone demonstrated more favorable prognoses. This study suggested that combining mutation data from the TERT promoter and IDH genes created a novel method of defining glioma subgroups to supplement the traditional histopathological criteria for disease diagnosis. To facilitate an increased use of these RNA sequencing data from the CGGA project, a free, web accessible and use-friendly database was also constructed (GLIOMASdb; http://cgga.org.cn:9091/gliomasdb/) (34). The GLIOMASdb currently provides data available to download and analysis of gene patterns in the malignant progression of gliomas.

Other datasets. The rapid expansion of integrated multi-omics, bioinformatics analyses and clinical translation has markedly altered the understanding of glioma. Over the previous decade, several other glioma genome projects have been undertaken.

The REpository of Molecular BRAin Neoplasia DaTa (REMBRANDT), a cancer clinical genomics database and an online mining and analysis platform, aimed to improve understanding of glioma by effectively combining clinical information and genomic characteristics (35). To date, REMBRANDT includes 874 glioma specimens comprising $\sim 566$ gene expression arrays, 834 DNA copy number arrays and 13,472 clinical data, which may be used as an independent dataset for glioma research.

To improve glioma stratification standards, Gravendeel et al (5) performed gene expression profiling (Affymetrix HU133 Plus 2.0, n=276) from patients with glioma (GSE16011). In this study, seven distinct molecular subgroups were identified and correlated with survival. These contained two subgroups with favorable prognosis (median survival $>4.7$ years), two intermediate prognostic subtypes (median survival of 1-4 years), two with poor prognosis (median survival of $<1$ year) and one control group. This result was validated by 5 other independent datasets, supporting this evidence that gene expression profiling may be an effective method to classify gliomas, and that this molecular classification may assist to diagnose and guide clinical decision making.

In addition, Ballester et al (36) performed a retrospective analysis of sequencing results of 381 primary gliomas. These cases, including GBM $(n=227)$, anaplastic astrocytomas $(n=46)$, diffuse astrocytoma $(n=37)$, anaplastic oligodendrogliomas $(n=21)$ and oligodendrogliomas $(n=11)$, were used to identify mutations and amplifications in cancer-associated genes using a validated, commercially-available panel. The results revealed that the most commonly mutated genes included TP53 (37.2\%), IDH1 (29.4\%), phosphatidylinositol-4,5-biphosphate 3-kinase catalytic subunit alpha (PIK3CA; 8\%), PTEN (8\%) and EGFR (7.5\%). In addition, 23\% cases (88/381) exhibited genomic amplification in at least 1 cancer-associated gene in the specific panel. The most common genes that indicated evidence of amplification included EGFR (18.0\%), PDGFRA (2.5\%) and KIT proto-oncogene receptor tyrosine kinase (1.8\%). This study demonstrated the utility of next-generation sequencing for the identification of genetic alterations in brain tumors in the clinical setting.

\section{Molecular profiling as a precise clinical tool}

At present, treatment decisions in patients with glioma primarily depend on histological classification and clinical parameters. However, the differences between histological subgroups and grades are subtle, and are susceptible to high inter-observer variability. Previously, additional data have suggested that the molecular profiles of gliomas are an improved predictor of survival compared with that of histology results (5). At present, most of studies have used information other than histological or clinical data to establish the molecular classifiers of glioma. The traditional molecular pathology of gliomas are summarized in Fig. 1. The key gene alternations in gliomas are summarized in Table II. 
IDH mutation and chromosome $1 p / 19 q$ codeletion. IDH1 and IDH2 are NADP+-dependent isocitrate dehydrogenases, catalyzing the oxidative decarboxylation of isocitrate to $\alpha$-ketoglutarate $(\alpha-\mathrm{KG})$ and converting NADP to NADPH. Previous data implied that the mutations in the IDH1 and IDH2 genes were closely associated with the pathogenesis of malignant gliomas $(18,37,38)$. Generally, patients with glioma with and without IDH mutations exhibit significantly different outcomes (12). In particular, IDH mutation, including R132H in the IDH1 gene, are commonly identified in WHO grade II and III disease (oligodendrogliomas, astrocytomas and secondary glioblastomas), suggesting that IDH mutations may be early events and promote the progression of gliomas (38). In addition, complete deletion of the short arm of chromosome 1 and the long arm of chromosome $19(1 \mathrm{p} / 19$ co-deletion) is the molecular genetic signature of oligodendrogliomas, which occurs early in its pathogenesis (39). The $1 \mathrm{p} / 19 \mathrm{q}$ co-deletion occurs in the majority of WHO grade II tumors, and has been a valuable diagnostic, prognostic and predictive biomarker for the management of oligodendroglial tumors (12).

TERT promoter mutations. TERT is a catalytic subunit of the telomerase and encodes a highly specialized reverse transcriptase. The role of TERT has been revealed by the frequent mutations of the TERT promoter (TERTp-mut) in gliomagenesis, but particularly in glioma $(12,18)$. The mutations of the TERT promoter frequently occur in 2 loci, C228T and C250T, mapping -124 and $-146 \mathrm{bp}$, respectively, upstream of the TERT ATG site. The presence of TERTp-mut, creating binding sites for E26 transformation-specific/T-cell factor transcription factors, is significantly associated with higher mRNA expression. Increasing evidence has suggested that TERTp-mut also affects cancer susceptibility and results in poorer prognosis for patients with glioma (40).

MGMT promoter methylation. DNA methylation of the gene promoter may serve an important role in carcinogenesis. MGMT, as a coding DNA repair gene, is crucial for genome stability. During DNA replication and transcription, the MGMT gene may repair the naturally occurring mutagenic DNA lesion O-6-methylguanine back to guanine, preventing mismatch and errors. A number of studies have demonstrated that the methylation state of the MGMT gene is closely associated with the response to temozolomide (41). Specifically, if the promoter is methylated, the treatment is more effective; otherwise, the patient is not sensitive, suggesting that MGMT promoter methylation is a favorable predictor for overall survival and progression-free survival in glioma $(42,43)$. At present, MGMT has also been revealed to be a useful tool for increasing gene therapy efficiency, and with applications in clinical detection (44).

Fusion genes. Gene rearrangements and the consequent fusion proteins serve an important role in tumorigenesis. It was previously demonstrated that a recurrent gene fusion event involving the protein tyrosine phosphatase receptor type Z1 (PTPRZ1) and MET proto-oncogene, receptor tyrosine kinase (MET) genes, termed the ZM fusion, was identified in $15 \%$ of secondary glioblastomas (45). In this fusion event, the PTPRZ1 promoter was activated to additionally promote the expression of full-length MET, leading to MET overexpression. Patients with ZM fusion exhibited more aggressive phenotypes. The recurrent nature of the ZM fusion suggests that $\mathrm{ZM}$ fusion is associated with GBM migration and invasion, participates in PIK3CA signaling, and results in a poorer prognosis, supporting $\mathrm{ZM}$ as a potential GBM therapeutic target. In addition, the fibroblast growth factor receptor (FGFR)-transforming acidic coiled-coil containing protein (TACC) was the first well-characterized gene fusion event in GBMs (46-48). These fusion proteins include the tyrosine kinase domain of FGFR and the coiled-coil domain of TACC proteins. There are 2 subtypes of the FGFR-TACC gene fusion protein, FGFR3-TACC3 (F3-T3) and FGFR3-TACC1, in GBMs. Previously, Bao et al (45) indicated that F3-T3 was associated with oxidative phosphorylation and mitochondrial biogenesis. FGFR-TACC gene fusion may also provide a promising target for GBM treatment.

\section{Conclusions}

Within the previous decade, the volume of newly-published biological data has grown rapidly, and has increased understanding of the key genomic alternations in human glioma. At present, several glioma-associated genomic studies have been initiated, including TCGA and the CGGA, aiming to generate and comprehensively describe the multi-dimensional glioma genome. These projects provided unprecedented and publicly available glioma datasets, used widely by the scientific community. To provide improved understanding of the genetics of glioma, several studies have classified glioma into subtypes and revealed their associations with clinical parameters, assisting in the generation of novel cancer therapies, diagnostic methods and preventive strategies.

Although malignant glioma remains incurable, treatment options have been expanding and improving due to improved understanding of the complex molecular biology of these tumors. Based on the 2016 WHO organization classification of brain tumors, diffuse gliomas are defined by histopathology and molecular pathology, particularly for molecular diagnosis. At present, several key genes in glioma have been used as biomarkers for predicting outcome and guiding chemoradiotherapy, including MGMT promoter methylation. As important biological molecules, non-coding RNAs are emerging as biomarkers or potential targets for glioma treatment (49-52). Future studies will also generate additional biological glioma data that may reveal novel biological markers as therapeutic targets/candidates in the treatment of tumors.

\section{Acknowledgements}

The authors would like to acknowledge Dr Fanlin Meng at the School of Medicine, Tsinghua University, Beijing, China for her assistance in revising the manuscript.

\section{Funding}

The present study was supported by The National Key Research and Development Plan (grant no. 2016YFC0902500). 


\section{Availability of data and materials}

Not applicable.

\section{Authors' contributions}

All authors participated in the design and finial review of the manuscript. JC and ZZ conceived the review. ZZ and $\mathrm{KZ}$ drafted the manuscript. ZW and KW collected the information on data resources. XL and FW collected the information on molecular profiles. All authors reviewed and approved the final manuscript.

\section{Ethics approval and consent to participate}

Not applicable.

\section{Patient consent for publication}

Not applicable.

\section{Competing interests}

The authors declare that they have no competing interests.

\section{References}

1. Omuro A and DeAngelis LM: Glioblastoma and other malignant gliomas: A clinical review. JAMA 310: 1842-1850, 2013.

2. Ohgaki H and Kleihues P: Epidemiology and etiology of gliomas. Acta Neuropathol 109: 93-108, 2005.

3. Ostrom QT, Gittleman H, Liao P, Vecchione-Koval T, Wolinsky Y, Kruchko C and Barnholtz-Sloan JS: CBTRUS Statistical Report: Primary brain and other central nervous system tumors diagnosed in the United States in 2010-2014. Neuro-oncol 19 (Suppl 5): v1-v88, 2017.

4. Louis DN, Perry A, Reifenberger G, von Deimling A, Figarella-Branger D, Cavenee WK, Ohgaki H, Wiestler OD, Kleihues P and Ellison DW: The 2016 World Health Organization Classification of Tumors of the Central Nervous System: A summary. Acta Neuropathol 131: 803-820, 2016.

5. Gravendeel LA, Kouwenhoven MC, Gevaert O, de Rooi JJ, Stubbs AP, Duijm JE, Daemen A, Bleeker FE, Bralten LB, Kloosterhof NK, et al: Intrinsic gene expression profiles of gliomas are a better predictor of survival than histology. Cancer Res 69: 9065-9072, 2009.

6. Buckner J, Giannini C, Eckel-Passow J, Lachance D, Parney I, Laack $\mathrm{N}$ and Jenkins R: Management of diffuse low-grade gliomas in adults - use of molecular diagnostics. Nat Rev Neurol 13: 340-351, 2017.

7. Zhao S, Cai J, Li J, Bao G, Li D, Li Y, Zhai X, Jiang C and Fan L: Bioinformatic Profiling Identifies a Glucose-Related Risk Signature for the Malignancy of Glioma and the Survival of Patients. Mol Neurobiol 54: 8203-8210, 2017.

8. Tomczak K, Czerwińska P and Wiznerowicz M: The Cancer Genome Atlas (TCGA): An immeasurable source of knowledge. Contemp Oncol (Pozn) 19 (1A): A68-A77, 2015.

9. Barretina J, Caponigro G, Stransky N, Venkatesan K, Margolin AA, Kim S, Wilson CJ, Lehár J, Kryukov GV, Sonkin D, et al: The Cancer Cell Line Encyclopedia enables predictive modelling of anticancer drug sensitivity. Nature 483: 603-607, 2012

10. Consortium EP; ENCODE Project Consortium: An integrated encyclopedia of DNA elements in the human genome. Nature 489: 57-74, 2012.

11. Arita H, Yamasaki K, Matsushita Y, Nakamura T, Shimokawa A, Takami H, Tanaka S, Mukasa A, Shirahata M, Shimizu S, et al: A combination of TERT promoter mutation and MGMT methylation status predicts clinically relevant subgroups of newly diagnosed glioblastomas. Acta Neuropathol Commun 4: 79, 2016.
12. Brat DJ, Verhaak RG, Aldape KD, Yung WK, Salama SR, Cooper LA, Rheinbay E, Miller CR, Vitucci M, Morozova O, et al; Cancer Genome Atlas Research Network: Comprehensive, Integrative Genomic Analysis of Diffuse Lower-Grade Gliomas. N Engl J Med 372: 2481-2498, 2015.

13. Verhaak RG, Hoadley KA, Purdom E, Wang V, Qi Y, Wilkerson MD, Miller CR, Ding L, Golub T, Mesirov JP, et al; Cancer Genome Atlas Research Network: Integrated genomic analysis identifies clinically relevant subtypes of glioblastoma characterized by abnormalities in PDGFRA, IDH1, EGFR, and NF1. Cancer Cell 17: 98-110, 2010.

14. Diamandis P and Aldape KD: Insights From Molecular Profiling of Adult Glioma. J Clin Oncol 35: 2386-2393, 2017.

15. Yang P, Cai J, Yan W, Zhang W, Wang Y, Chen B, Li G, Li S, Wu C, Yao K, et al; CGGA project: Classification based on mutations of TERT promoter and IDH characterizes subtypes in grade II/III gliomas. Neuro-oncol 18: 1099-1108, 2016.

16. Yang P, Cai J, Yan W, Zhang W, Wang Y, Chen B, Li G, Li S, Wu Yao K, et al; CGGA project: Classification based on mutations of TERT promoter and IDH characterizes subtypes in grade II/III gliomas. Neuro-oncol 18: 1099-1108, 2016.

17. Huang L, Jiang T, Yuan F, Li GL, Cui Y, Liu EZ and Wang ZC: Correlation of chromosomes $1 \mathrm{p}$ and $19 \mathrm{q}$ status and expressions of O6-methylguanine DNA methyltransferase (MGMT), p53 and $\mathrm{Ki}-67$ in diffuse gliomas of World Health Organization (WHO) grades II and III: A clinicopathological study. Neuropathol Appl Neurobiol 35: 367-379, 2009.

18. Eckel-Passow JE, Lachance DH, Molinaro AM, Walsh KM, Decker PA, Sicotte H, Pekmezci M, Rice T, Kosel ML, Smirnov IV, et al: Glioma Groups Based on 1p/19q, IDH, and TERT Promoter Mutations in Tumors. N Engl J Med 372: 2499-2508, 2015.

19. Killela PJ, Reitman ZJ, Jiao Y, Bettegowda C, Agrawal N, DiazLA Jr, Friedman AH, Friedman H, Gallia GL, Giovanella BC, et al: TERT promoter mutations occur frequently in gliomas and a subset of tumors derived from cells with low rates of self-renewal. Proc Natl Acad Sci USA 110: 6021-6026, 2013

20. Pekmezci M, Rice T, Molinaro AM, Walsh KM, Decker PA, Hansen H, Sicotte H, Kollmeyer TM, McCoy LS, Sarkar G, et al: Adult infiltrating gliomas with WHO 2016 integrated diagnosis: Additional prognostic roles of ATRX and TERT. Acta Neuropathol 133: 1001-1016, 2017.

21. Wang SI, Puc J, Li J, Bruce JN, Cairns P, Sidransky D and Parsons R: Somatic mutations of PTEN in glioblastoma multiforme. Cancer Res 57: 4183-4186, 1997.

22. Koschmann C, Calinescu AA, Nunez FJ, Mackay A, Fazal-Salom J, Thomas D, Mendez F, Kamran N, Dzaman M, Mulpuri L, et al: ATRX loss promotes tumor growth and impairs nonhomologous end joining DNA repair in glioma. Sci Transl Med 8: 328ra28, 2016.

23. Dahiya S, Emnett RJ, Haydon DH, Leonard JR, Phillips JJ, Perry A and Gutmann DH: BRAF-V600E mutation in pediatric and adult glioblastoma. Neuro-oncol 16: 318-319, 2014.

24. Wick W, Weller M, van den Bent M, Sanson M, Weiler M, von Deimling A, Plass C, Hegi M, Platten $M$ and Reifenberger G: MGMT testing - the challenges for biomarker-based glioma treatment. Nat Rev Neurol 10: 372-385, 2014.

25. Frattini V, Trifonov V, Chan JM, Castano A, Lia M, Abate F, Keir ST, Ji AX, Zoppoli P, Niola F, et al: The integrated landscape of driver genomic alterations in glioblastoma. Nat Genet 45: $1141-1149,2013$.

26. No authors listed. The future of cancer genomics. Nat Med 21: 99, 2015.

27. Stratton MR, Campbell PJ and Futreal PA: The cancer genome. Nature 458: 719-724, 2009.

28. Brennan CW, VerhaakRG, McKenna A, Campos B, NoushmehrH, Salama SR, Zheng S, Chakravarty D, Sanborn JZ, Berman SH, et al; TCGA Research Network: The somatic genomic landscape of glioblastoma. Cell 155: 462-477, 2013.

29. Lee JK, Wang J, Sa JK, Ladewig E, Lee HO, Lee IH, Kang HJ, Rosenbloom DS, Camara PG, Liu Z, et al: Spatiotemporal genomic architecture informs precision oncology in glioblastoma. Nat Genet 49: 594-599, 2017.

30. Cancer Genome Atlas Research Network: Comprehensive genomic characterization defines human glioblastoma genes and core pathways. Nature 455: 1061-1068, 2008.

31. Cerami E, Gao J, Dogrusoz U, Gross BE, Sumer SO, Aksoy BA, Jacobsen A, Byrne CJ, Heuer ML, Larsson E, et al: The cBio cancer genomics portal: An open platform for exploring multidimensional cancer genomics data. Cancer Discov 2: 401-404, 2012. 
32. Gao J, Aksoy BA, Dogrusoz U, Dresdner G, Gross B, Sumer SO, Sun Y, Jacobsen A, Sinha R, Larsson E, et al: Integrative analysis of complex cancer genomics and clinical profiles using the cBioPortal. Sci Signal 6: pl1, 2013.

33. Yan W, Zhang W, You G, Zhang J, Han L, Bao Z, Wang Y, Liu Y, Jiang C, Kang C, et al: Molecular classification of gliomas based on whole genome gene expression: A systematic report of 225 samples from the Chinese Glioma Cooperative Group. Neuro-oncol 14: 1432-1440, 2012.

34. Zhao Z, Meng F, Wang W, Wang Z, Zhang C and Jiang T: Comprehensive RNA-seq transcriptomic profiling in the malignant progression of gliomas. Sci Data 4: 170024, 2017.

35. Madhavan S, Zenklusen JC, Kotliarov Y, Sahni H, Fine HA and Buetow K: Rembrandt: Helping personalized medicine become a reality through integrative translational research. Mol Cancer Res 7: 157-167, 2009.

36. Ballester LY, Fuller GN, Powell SZ, Sulman EP, Patel KP, Luthra R and Routbort MJ: Retrospective Analysis of Molecular and Immunohistochemical Characterization of 381 Primary Brain Tumors. J Neuropathol Exp Neurol 76: 179-188, 2017.

37. Ducray F, Marie Y and Sanson M: IDH1 and IDH2 mutations in gliomas. N Engl J Med 360: 2248-2249, author reply 2249, 2009

38. Yan H, Parsons DW, Jin G, McLendon R, Rasheed BA, Yuan W, Kos I, Batinic-Haberle I, Jones S, Riggins GJ, et al: IDH1 and IDH2 mutations in gliomas. N Engl J Med 360: 765-773, 2009.

39. Vijayakumar V, Liebisch G, Buer B, Xue L, Gerlach N, Blau S, Schmitz J and Bucher M: Integrated multi-omics analysis supports role of lysophosphatidylcholine and related glycerophospholipids in the Lotus japonicus-Glomus intraradices mycorrhizal symbiosis. Plant Cell Environ 39: 393-415, 2016

40. Diplas BH, He X, Brosnan-Cashman JA, Liu H, Chen LH, Wang Z, Moure CJ, Killela PJ, Loriaux DB, Lipp ES, et al: The genomic landscape of TERT promoter wildtype-IDH wildtype glioblastoma. Nat Commun 9: 2087, 2018.

41. Perry JR, Laperriere N, O'Callaghan CJ, Brandes AA, Menten J, Phillips C, Fay M, Nishikawa R, Cairncross JG, Roa W, et al, Trial Investigators: Short-Course Radiation plus Temozolomide in Elderly Patients with Glioblastoma. N Engl J Med 376 : 1027-1037, 2017

42. Weller M, Stupp R, Reifenberger G, Brandes AA, van den Bent MJ, Wick W and Hegi ME: MGMT promoter methylation in malignant gliomas: Ready for personalized medicine? Nat Rev Neurol 6: 39-51, 2010

43. Petrova RS, Webb KF, Vaghefi E, Walker K, Schey KL and Donaldson PJ: Dynamic functional contribution of the water channel AQP5 to the water permeability of peripheral lens fiber cells. Am J Physiol Cell Physiol 314: C191-C201, 2018.

44. Binabaj MM, Bahrami A, ShahidSales S, Joodi M Joudi Mashhad M, Hassanian SM, Anvari K and Avan A: The prognostic value of MGMT promoter methylation in glioblastoma: A meta-analysis of clinical trials. J Cell Physiol 233: 378-386, 2018
45. Bao ZS, Chen HM, Yang MY, Zhang CB, Yu K, Ye WL, Hu BQ, Yan W, Zhang W, Akers J, et al: RNA-seq of 272 gliomas revealed a novel, recurrent PTPRZ1-MET fusion transcript in secondary glioblastomas. Genome Res 24: 1765-1773, 2014.

46. Singh D, Chan JM, Zoppoli P, Niola F, Sullivan R, Castano A, Liu EM, Reichel J, Porrati P, Pellegatta S, et al: Transforming fusions of FGFR and TACC genes in human glioblastoma. Science 337: 1231-1235, 2012.

47. Di Stefano AL, Fucci A, Frattini V, Labussiere M, Mokhtari K, Zoppoli P, Marie Y, Bruno A, Boisselier B, Giry M, et al: Detection, Characterization, and Inhibition of FGFR-TACC Fusions in IDH Wild-type Glioma. Clin Cancer Res 21: 3307-3317, 2015

48. Lasorella A, Sanson M and Iavarone A: FGFR-TACC gene fusions in human glioma. Neuro Oncol 19: 475-483, 2017.

49. Li Y, Xu J, Chen H, Bai J, Li S, Zhao Z, Shao T, Jiang T, Ren $\mathrm{H}$, Kang C, et al: Comprehensive analysis of the functional microRNA-mRNA regulatory network identifies miRNA signatures associated with glioma malignant progression. Nucleic Acids Res 41: e203, 2013.

50. Luo H, Chen Z, Wang S, Zhang R, Qiu W, Zhao L, Peng C, Xu R, Chen W, Wang HW, et al: c-Myc-miR-29c-REV3L signalling pathway drives the acquisition of temozolomide resistance in glioblastoma. Brain 138: 3654-3672, 2015.

51. Wang Z, Zhang C, Liu X, Wang Z, Sun L, Li G, Liang J, Hu H, Liu Y, Zhang W, et al: Molecular and clinical characterization of PD-L1 expression at transcriptional level via 976 samples of brain glioma. OncoImmunology 5: e1196310, 2016.

52. Zhang CB, Zhu P, Yang P, Cai JQ, Wang ZL, Li QB, Bao ZS, Zhang $\mathrm{W}$ and Jiang T: Identification of high risk anaplastic gliomas by a diagnostic and prognostic signature derived from mRNA expression profiling. Oncotarget 6: 36643-36651, 2015.

53. Lee Y, Scheck AC, Cloughesy TF, Lai A, Dong J, Farooqi HK, Liau LM, Horvath S, Mischel PS and Nelson SF: Gene expression analysis of glioblastomas identifies the major molecular basis for the prognostic benefit of younger age. BMC Med Genomics 1: 52,2008 .

54. Phillips HS, Kharbanda S, Chen R, Forrest WF, Soriano RH, Wu TD, Misra A, Nigro JM, Colman H, Soroceanu L, et al: Molecular subclasses of high-grade glioma predict prognosis, delineate a pattern of disease progression, and resemble stages in neurogenesis. Cancer Cell 9: 157-173, 2006. 